This is a self-archived version of an original article. This version may differ from the original in pagination and typographic details.

Author(s): Heßberger, F. P.; Block, M.; Düllmann, Ch. E.; Yakushev, A.; Leino, M.; Uusitalo, J.

Title: Some Remarks on the Discovery of 244Md

Year: 2021

Version: Published version

Copyright: ㄷ 2021 APS

Rights: In Copyright

Rights url: http://rightsstatements.org/page//nC/1.0/?language=en

Please cite the original version:

Heßberger, F. P., Block, M., Düllmann, Ch. E., Yakushev, A., Leino, M., \& Uusitalo, J. (2021). Some Remarks on the Discovery of 244Md. Physical Review Letters, 126(18), Article 182501.

https://doi.org/10.1103/PhysRevLett.126.182501 


\title{
Some Remarks on the Discovery of ${ }^{244} \mathrm{Md}$
}

\author{
F. P. Heßberger®, ${ }^{1,2}$ M. Block ${ }^{1,2,3}$ Ch. E. Düllmann, ${ }^{1,2,3}$ A. Yakushev $\odot,{ }^{2}$ M. Leino $\odot,{ }^{4}$ and J. Uusitalo ${ }^{4}$ \\ ${ }^{1}$ Helmholtz-Institut Mainz, 55099 Mainz, Germany \\ ${ }^{2}$ GSI Helmholtzzentrum für Schwerionenforschung, 64291 Darmstadt, Germany \\ ${ }^{3}$ Johannes Gutenberg-Universität Mainz, 55099 Mainz, Germany \\ ${ }^{4}$ University Jyväskylä, 40014 Jyväskylä, Finland
}

(Received 20 November 2020; accepted 2 April 2021; published 3 May 2021)

\begin{abstract}
In two recent papers by Pore et al. and Khuyagbaatar et al., discovery of the new isotope ${ }^{244} \mathrm{Md}$ was reported. The decay data, however, are conflicting. While Pore et al. report two isomeric states decaying by $\alpha$ emission with $E_{\alpha}(1)=8.66(2) \mathrm{MeV}, T_{1 / 2}(1)=0.4_{-0.1}^{+0.4} \mathrm{~s}$ and $E_{\alpha}(2)=8.31(2) \mathrm{MeV}, T_{1 / 2}(2) \approx 6 \mathrm{~s}$, Khuyagbaatar et al. [Phys. Rev. Lett. 125, 142504 (2020).] report only a single transition with a broad energy distribution of $E_{\alpha}=(8.73-8.86) \mathrm{MeV}$ and $T_{1 / 2}=0.30_{-0.09}^{+0.19} \mathrm{~s}$. The data published in Pore et al. are very similar to those published for ${ }^{245 m} \mathrm{Md}\left[E_{\alpha}=8.64(2), 8.68(2) \mathrm{MeV}, T_{1 / 2}=0.35_{-0.16}^{+0.23} \mathrm{~s}\right.$ [V. Ninov, F. P. Heßberger, S. Hofmann, H. Folger, G. Münzenberg, P. Armbruster, A. V. Yeremin, A. G. Popeko, M. Leino, and S. Saro, Z. Phys. A 356, 11 (1996).] ]. Therefore, we compare the data presented for ${ }^{244} \mathrm{Md}$ in Pore et al. with those reported for ${ }^{245} \mathrm{Md}$ in Ninov et al. and also in Khuyagbaatar et al. We conclude that the data presented in Pore et al. shall be attributed to ${ }^{245} \mathrm{Md}$ with small contributions (one event each) from ${ }^{245} \mathrm{Fm}$ and probably ${ }^{246} \mathrm{Md}$.
\end{abstract}

DOI: $10.1103 /$ PhysRevLett.126.182501

Introduction.-Discovery of ${ }^{244} \mathrm{Md}$ was first reported by Pore et al. [1]. They used the reaction ${ }^{209} \mathrm{Bi}\left({ }^{40} \mathrm{Ar}, 5 \mathrm{n}\right){ }^{244} \mathrm{Md}$ at a bombarding energy of $\approx 220 \mathrm{MeV}$, which corresponds to an excitation energy of the compound nucleus ${ }^{249} \mathrm{Md}$ of $E^{*} \approx 46 \mathrm{MeV}$ at a production in the center of the target. They observed four events after the mass spectrometer FIONA at a position where events with mass number $A=$ 244 were expected, and six $\alpha$ decay chains in the BGS focal plane detector. The latter were attributed to the decay of two states in ${ }^{244} \mathrm{Md}$, one with $E_{\alpha}=8.308 \pm 0.019 \mathrm{MeV}, T_{1 / 2} \approx$ $6 \mathrm{~s}$ ( 1 event), and one with $E_{\alpha}=8.663 \pm 0.023 \mathrm{MeV}$, $T_{1 / 2}=0.4_{-0.1}^{+0.4} \mathrm{~s} \quad(4$ events $)$. In a publication by Khuyagbaatar et al., identification of ${ }^{244} \mathrm{Md}$ was reported using the reaction ${ }^{197} \mathrm{Au}\left({ }^{50} \mathrm{Ti}, 3 \mathrm{n}\right){ }^{244} \mathrm{Md}$ [2]. The experiment was performed at two bombarding energies of 239.8 and $231.5 \mathrm{MeV}$ (center of target), corresponding to excitation energies of $E^{*}=32.7$ and $E^{*}=26.2 \mathrm{MeV}$. They reported two $\alpha$ activities. One, with an energy range $E_{\alpha}=(8.7-8.9) \mathrm{MeV}$ and a half-life of $T_{1 / 2}=0.30_{-0.09}^{+0.19} \mathrm{~s}$ was observed only at the higher excitation energy (7 events); the second activity was observed at both energies (three events each with full energy release in the stop detector) within an energy range of $E_{\alpha}=(8.6-8.7) \mathrm{MeV}$ and a half-life of $T_{1 / 2}=0.33_{-0.08}^{+0.15} \mathrm{~s}$. This activity was attributed to the previously reported isotope ${ }^{245} \mathrm{Md}$. The isotope ${ }^{245} \mathrm{Md}$ was first observed in an experiment performed at the velocity filter SHIP at GSI, Darmstadt, Germany, using the reaction ${ }^{209} \mathrm{Bi}\left({ }^{40} \mathrm{Ar}, 4 \mathrm{n}\right){ }^{245} \mathrm{Md}$ at a bombarding energy of $5.12 \mathrm{AMeV}(204.8 \mathrm{MeV})$ corresponding to an excitation energy of $E^{*}=40 \mathrm{MeV}$ [3].
The authors reported two $\alpha$ energies of $E_{\alpha}=8640 \pm 20$, $8680 \pm 20 \mathrm{keV}$, and a half-life of $T_{1 / 2}=0.35_{-0.18}^{+0.23} \mathrm{~s}$ and also a spontaneous fission activity of $T_{1 / 2}=0.90_{-0.16}^{+0.23} \mathrm{~ms}$. This fission activity with $T_{1 / 2}=0.9_{-0.3}^{+0.6} \mathrm{~ms}$ was also observed by Khuyagbaatar et al. [2]. The fission activity was attributed to the ground state decay of ${ }^{245} \mathrm{Md}$, and the $\alpha$ activity to an isomeric state ${ }^{245 m} \mathrm{Md}$ [3]. Previously known data on ${ }^{245} \mathrm{Md}$ were not mentioned in Ref. [1]. For completeness it should be noted that on the basis of detailed spectroscopic investigation of odd-mass mendelevium isotopes performed since then [4], the $\alpha$ activity would nowadays rather be attributed to ${ }^{245} \mathrm{Md}$ and the fission activity to ${ }^{245 m} \mathrm{Md}$. It further was shown in Ref. [4] that $\alpha$ decay in odd mass mendelevium isotopes populates predominantly the $7 / 2^{-}[514]$ Nilsson level in the einsteinium daughter nuclei which decay into the $7 / 2^{+}[633]$ Nilsson level and the $9 / 2^{+}$member of the rotational band built up on it. As the $9 / 2^{+}$level decays by highly converted M1 transitions into the $7 / 2^{+}$band head, the line at $E_{\alpha}=8680 \pm 20 \mathrm{keV}$ reported in Ref. [3] is thus certainly the result of energy summing of $\alpha$ particles and conversion electrons.

Comparison of the results for ${ }^{245} \mathrm{Md}$ reported by Ninov et al. [3] and Khuyagbaatar et al. [2] and for ${ }^{244} \mathrm{Md}$ reported by Pore et al. [1].- The data published for ${ }^{245} \mathrm{Md}$ in Refs. [2,3] and ${ }^{244} \mathrm{Md}$ in Ref. [1] are presented in Fig. 1 and Table I. Data of Pore et al. (P1-P6) are taken from Table I in Ref. [1]. Data of Khuyagbataar et al. (K1-K10) are taken from the Supplemental Material of Ref. [2]. 


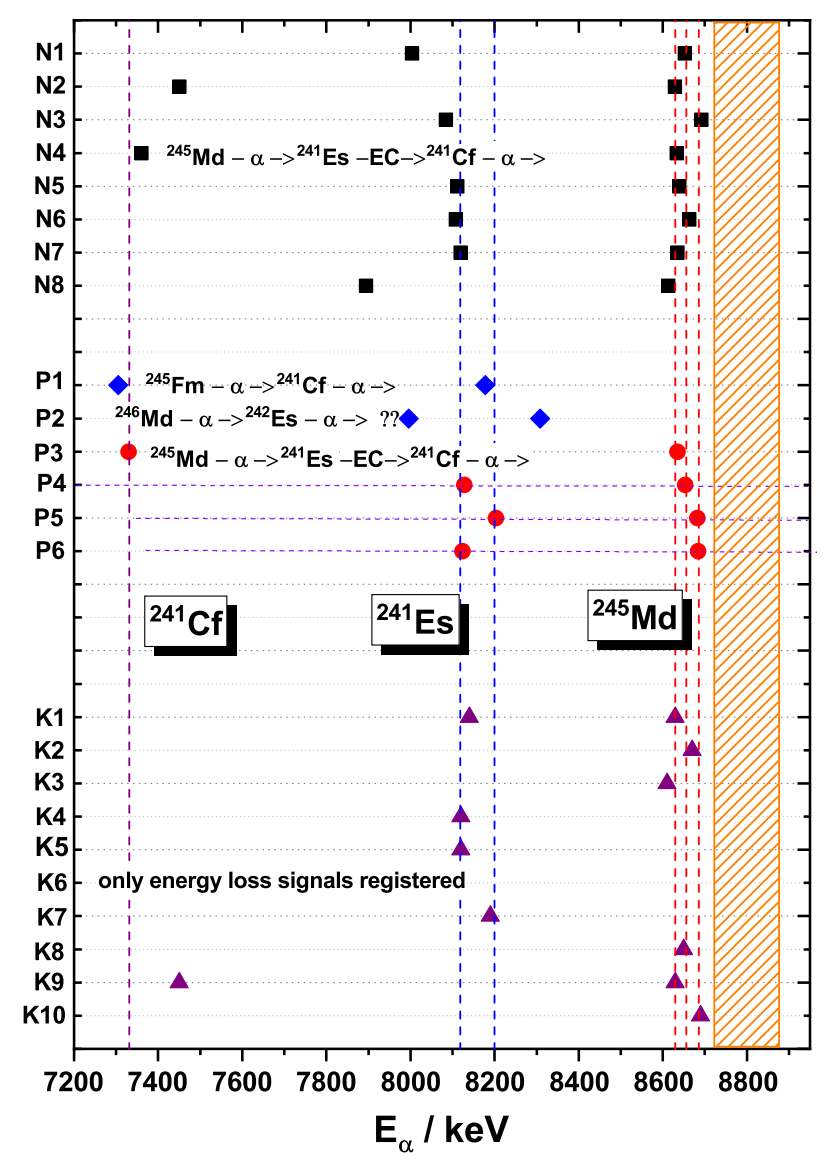

FIG. 1. Summary of decays attributed to ${ }^{245} \mathrm{Md}$ in Ref. [3] (squares) as well as in Ref. [2] (triangles) together with data reported by Pore et al. [1] [circles: events attributed to ${ }^{245} \mathrm{Md}$ by the present authors, diamonds: events attributed to ${ }^{245} \mathrm{Fm}$ or (tentatively) to ${ }^{246} \mathrm{Md}$ ]. The dashed lines are to guide the eyes: the red lines represent the $\alpha$ energies given for ${ }^{245} \mathrm{Md}$ (8640, $8680 \mathrm{keV})$ in Ref. [3] and the energy given for ${ }^{244} \mathrm{Md}$ (8663 keV) in Ref. [1]; the blue lines represent the $\alpha$ energy for ${ }^{241}$ Es $(8113 \mathrm{keV})$ given in Ref. [3] and the highest daughter energy (P5) in Ref. [1]; the purple line represents the literature value of the $\alpha$ energy of ${ }^{241} \mathrm{Cf}(7335 \mathrm{keV})$ [6]. The orange hatched area marks the range of $\alpha$ energies where the events attributed to ${ }^{244} \mathrm{Md}$ in Ref. [2] were observed.

No list of single events was presented by Ninov et al. [3]. Data shown here $(\mathrm{N} 1-\mathrm{N} 8)$ are taken from a reinspection of the logbook of the corresponding SHIP experiment [5]. Only $\alpha-\alpha$ correlations with full energy release of both $\alpha$ particles in the SHIP "stop detector" are listed.

Evidently the chains P3, P4, and P6 agree with the data reported for ${ }^{245} \mathrm{Md}$ in Refs. [2,3]. The energy of the daughter in P5 is higher than the values reported for ${ }^{241}$ Es in Ref. [3], but is in agreement with the daughter energy in K7. This event was attributed to ${ }^{245} \mathrm{Md}$ in Ref. [2] as it was registered at $E^{*}=26.2 \mathrm{MeV}$, where only decays of ${ }^{245} \mathrm{Md}$ were observed. Concerning the daughter energies P4, P5, and P6 can be attributed to the decay ${ }^{245} \mathrm{Md} \stackrel{\alpha}{\rightarrow}{ }^{241} \mathrm{Es} \stackrel{\alpha}{\rightarrow}$, while P3 obviously represents the decay ${ }^{245} \mathrm{Md} \stackrel{\alpha}{\rightarrow}{ }^{241} \mathrm{Es} \stackrel{E C}{\rightarrow}{ }^{241} \mathrm{Cf} \stackrel{\alpha}{\rightarrow}$, in
TABLE I. Summary of decays attributed to ${ }^{245} \mathrm{Md}$ in Refs. [2,3] and decays reported by Pore et al. [1]. Data from Pore et al. are taken from Table I in Ref. [1]; data from Khuyagbaatar et al. are from the Supplemental Material of Ref. [2]. No individual decay data are reported in Ref. [3]; these data are taken from the experiment analysis logbook [5].

\begin{tabular}{|c|c|c|c|c|c|}
\hline References & $\begin{array}{c}\text { Event } \\
\text { number }\end{array}$ & $\begin{array}{c}E_{\alpha}(1) / \\
\mathrm{MeV}\end{array}$ & $\Delta t(\mathrm{ER}-\alpha 1) / \mathrm{s}$ & $\begin{array}{c}E_{\alpha}(2) / \\
\mathrm{MeV}\end{array}$ & $\Delta \mathrm{t}(\alpha 1-\alpha 2) / \mathrm{s}$ \\
\hline [3] & N1 & 8.652 & 0.0178 & 8.004 & 8.254 \\
\hline [3] & $\mathrm{N} 2^{\mathrm{a}}$ & 8.629 & 0.1751 & 7.450 & 88.083 \\
\hline [3] & N3 & 8.692 & 0.00164 & 8.084 & 28.406 \\
\hline [3] & N4 & 8.633 & 0.1565 & 7.360 & 203.876 \\
\hline [3] & N5 & 8.639 & 1.1708 & 8.111 & 7.639 \\
\hline [3] & N6 & 8.663 & 0.0843 & 8.108 & 15.763 \\
\hline [3] & N7 & 8.635 & 0.2831 & 8.119 & 13.573 \\
\hline [3] & N8 & 8.613 & 0.0914 & 7.894 & 335.005 \\
\hline [2] & $\mathrm{K} 1^{\mathrm{b}}$ & 8.63 & 0.564 & 8.14 & 4.73 \\
\hline [2] & $\mathrm{K} 2^{\mathrm{b}}$ & 8.67 & 0.454 & (1.1) & 0.24 \\
\hline [2] & $\mathrm{K} 3^{\mathrm{b}}$ & 8.61 & 0.423 & (1.3) & 2.86 \\
\hline [2] & $\mathrm{K} 4^{\mathrm{b}}$ & (1.9) & 0.120 & 8.12 & 6.87 \\
\hline [2] & $\mathrm{K} 5^{\mathrm{b}}$ & $(2.2)$ & 0.508 & 8.12 & 11.5 \\
\hline [2] & $\mathrm{K} 6^{\mathrm{b}}$ & $(0.9)$ & 0.131 & 8.09 & 15.1 \\
\hline [2] & $\mathrm{K} 7^{\mathrm{b}}$ & $(0.4)$ & 1.42 & 8.19 & 2.97 \\
\hline [2] & $K 8^{\mathrm{c}}$ & 8.65 & 0.693 & $(0.26)$ & 5 \\
\hline [2] & $K 9^{c}$ & 8.63 & 0.346 & 7.45 & 20 \\
\hline [2] & $\mathrm{K} 10^{\mathrm{c}}$ & 8.69 & 0.129 & missed & missed \\
\hline [1] & P1 & 8.178 & 0.60 & 7.305 & 27.34 \\
\hline [1] & $\mathrm{P} 2$ & 8.308 & 9.18 & 7.996 & 14.37 \\
\hline [1] & P3 & 8.635 & 0.88 & 7.330 & 18.95 \\
\hline [1] & $P 4^{\mathrm{d}}$ & 8.653 & 0.13 & 8.128 & 1.20 \\
\hline [1] & P5 & 8.682 & 0.31 & 8.203 & 10.00 \\
\hline [1] & P6 & 8.684 & 1.16 & 8.124 & 7.65 \\
\hline
\end{tabular}

${ }^{\mathrm{a}}$ Both events were registered within the beam on period.

${ }^{\mathrm{b}}$ Observed at $E^{*}=26.2 \mathrm{MeV}$.

${ }^{c}$ Observed at $E^{*}=32.7 \mathrm{MeV}$.

${ }^{\mathrm{d}}$ The $\alpha-\alpha$ correlation was followed by a third event of $E_{\alpha}=$ $7.086 \pm 25 \mathrm{MeV}$ after $\Delta \mathrm{t}=75.97 \mathrm{~s}$.

accordance with $\mathrm{N} 4$ and the known $\alpha$ decay energy of ${ }^{241} \mathrm{Cf}$ (7.335 MeV [6]). P1 fits to the decay sequence ${ }^{245} \mathrm{Fm} \stackrel{\alpha(8.15 \mathrm{MeV})}{\rightarrow}{ }^{241} \mathrm{Cf} \stackrel{\alpha(8.34 \mathrm{MeV})}{\rightarrow}[6]$, with ${ }^{245} \mathrm{Fm}$ being the product of the $p 3 n$ channel. The cross-section ratio $\sigma(p 3 n) / \sigma(4 n) \approx 0.25$ may appear unusually high, but it has to be considered that one approaches the proton drip line, and proton binding energies are already low. The mass evaluation of Wang et al. [7] delivers values of, e.g., $1540 \pm$ $210 \mathrm{keV}$ for ${ }^{247} \mathrm{Md}$ and $1360 \pm 320 \mathrm{keV}$ for ${ }^{246} \mathrm{Md}$, significantly lower than the neutron binding energies of $8250 \pm$ $330 \mathrm{keV}\left({ }^{247} \mathrm{Md}\right)$ and $7230 \pm 400 \mathrm{keV}\left({ }^{246} \mathrm{Md}\right)$. And indeed HIVAP calculations [8] deliver even a ratio $\sigma(p 3 n) / \sigma(4 n) \approx$ 0.5 (see Fig. 2). It should be reminded that recently notable cross sections for $p$-evaporation channels have been reported for the reaction ${ }^{50} \mathrm{Ti}+{ }^{209} \mathrm{Bi}[9,10]$. Less clear is chain $\mathrm{P} 2$. The decay sequence ${ }^{246} \mathrm{Md} \stackrel{\alpha}{\rightarrow}{ }^{242} \mathrm{Es} \stackrel{\alpha}{\rightarrow}$, for which very broad energy distributions in the range $E_{\alpha} \approx(8.15-8.75) \mathrm{MeV}$ 


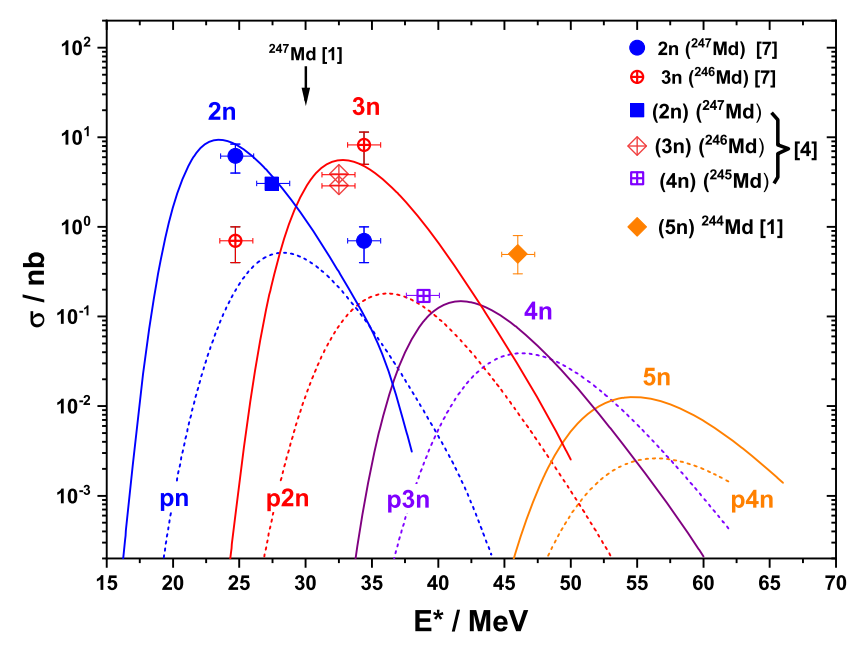

FIG. 2. Excitation function for ${ }^{40} \mathrm{Ar}+{ }^{209} \mathrm{Bi}$. The energies refer to production in the center of the target. The error bars for the energies refer to the energy loss of ${ }^{40} \mathrm{Ar}$ ions in the bismuth targets [12]. Systematic errors in the accelerator energy are typically $0.2 \%$ for the UNILAC accelerator and are neglected. For the data of Pore et al. [1] an energy loss of $\approx 12.5 \mathrm{MeV}$ in the titanium backing foil [12] is considered. No systematic error for the accelerator energy is given by Pore et al. Lines are the result of HIVAP [8] calculations; full lines represent $x n$ channels, dashed lines represent $p x n$ channels. Points are defined in the figure. The arrow marks the energy reported in Ref. [1] for the observation of ${ }^{247} \mathrm{Md}$.

$\left({ }^{246} \mathrm{Md}\right)$ and $E_{\alpha} \approx(7.75-8.05) \mathrm{MeV}\left({ }^{242} \mathrm{Es}\right)$ were observed (see Fig. 5 in Ref. [11]) is a possible candidate. P4 is terminated by an $\alpha$ event of $E_{\alpha}=7.086 \pm 0.025 \mathrm{MeV}$, which could be attributed to ${ }^{237} \mathrm{Bk}$, the so far unknown $\alpha$ daughter of ${ }^{241}$ Es. From atomic mass extrapolation [7] one expects an $\alpha$ decay energy of $E=7.376 \pm 0.242 \mathrm{MeV}$. The lower value could be due to the population of an excited state in ${ }^{233} \mathrm{Am}$.

Excitation functions.-The reported cross sections for production of ${ }^{244-247} \mathrm{Md}$ in the reaction ${ }^{209} \mathrm{Bi}\left({ }^{40} \mathrm{Ar}, \mathrm{xn}\right){ }^{249-x} \mathrm{Md}[1,5,11]$ are shown in Fig. 2. In Ref. [3] no cross sections are given. The values given for this experiment are taken from Ref. [5]. The lines are the result of HIVAP [8] calculations, using fission barriers modified to reproduce the $2 n\left({ }^{247} \mathrm{Md}\right)$ and $3 n\left({ }^{246} \mathrm{Md}\right)$ cross sections. Evidently the $4 n$ cross section from Ref. [5] is reproduced quite well. The excitation energy given by
Pore et al. [1] appears roughly $4 \mathrm{MeV}$ above the expected maximum for the $4 n$ cross section, and the value is about a factor of 6 higher, but more than 2 orders of magnitude higher than the value expected for the $5 n$ channel. A similiar situation is evident for the $2 n$ channel. Pore et al. [1] report the observation of ${ }^{247} \mathrm{Md}$ at a bombarding energy of $200 \mathrm{MeV}$, which corresponds to an excitation energy $E^{*} \approx 30 \mathrm{MeV}$ (arrow in Fig. 2), which is about $6 \mathrm{MeV}$ above the expected maximum for the $2 n$ channel, but still a notable production cross section of $\approx 2$ nbarn is expected here. To conclude, comparison with reported cross sections for $x n$ channels and HIVAP calculations indicates that the events attributed to ${ }^{244} \mathrm{Md}$ in Ref. [1] may rather stem from decay of ${ }^{245} \mathrm{Md}$.

Conclusion.-The decay data for ${ }^{244} \mathrm{Md}$ presented by Pore et al. [1] are in disagreement with those published by Khuyagbaatar et al. [2]. A critical inspection of the decay data of Pore et al. [1] for ${ }^{244} \mathrm{Md}$ and a comparison with reported decay data for ${ }^{245} \mathrm{Md}$ rather suggest that they have observed ${ }^{245} \mathrm{Md}$. An additional argument supporting that interpretation comes from the excitation function for the production of mendelevium isotopes in the reaction ${ }^{40} \mathrm{Ar}+{ }^{209} \mathrm{Bi}$. The excitation energy given for the observation of ${ }^{244} \mathrm{Md}$ is about $10 \mathrm{MeV}$ lower than the expected maximum for the $5 n$ channel. Bombarding energy and reported production cross section rather hint at the synthesis of ${ }^{245} \mathrm{Md}$.

[1] J. L. Pore et al., Phys. Rev. Lett. 124, 252502 (2020).

[2] J. Khuyagbaatar et al., Phys. Rev. Lett. 125, 142504 (2020).

[3] V. Ninov, F. P. Heßberger, S. Hofmann, H. Folger, G. Münzenberg, P. Armbruster, A. V. Yeremin, A. G. Popeko, M. Leino, and S. Saro, Z. Phys. A 356, 11 (1996).

[4] F. P. Heßberger et al., Eur. Phys. J. A 26, 233 (2005).

[5] F. P. Heßberger, Analysis Logbook SHIP Experiment R165, 1993 (to be published).

[6] R. B. Firestone et al., Table of Isotopes, 8th ed. (John Wiley \& Sons, New York, 1996).

[7] M. Wang et al., Chin. Phys. C 41, 030003 (2016).

[8] W. Reisdorf and M. Schädel, Z. Phys. A 343, 47 (1992).

[9] A. Lopez-Martens et al., Phys. Lett. B 795, 271 (2019).

[10] F. P. Heßberger, Eur. Phys. J. A 55, 208 (2019).

[11] S. Antalic et al., Eur. Phys. J. A 43, 35 (2010).

[12] J. F. Ziegler, J. P. Biersack, and M. D. Ziegler, SRIM2013.00, http://srim.org/ (2013). 